\title{
CHANDRA DETECTION OF A NEW DIFFUSE X-RAY COMPONENT FROM THE GLOBULAR CLUSTER 47 TUCANAE
}

\author{
E. M. H. Wu ${ }^{1}$, C. Y. HuI ${ }^{2}$, A. K. H. Kong ${ }^{3}$, P. H. T. TAM ${ }^{3}$, K. S. Cheng ${ }^{1}$, And V. A. DogIEL ${ }^{4}$ \\ ${ }^{1}$ Department of Physics, University of Hong Kong, Pokfulam Road, Hong Kong \\ ${ }^{2}$ Department of Astronomy and Space Science, Chungnam National University, Daejeon, Korea; cyhui@ @nu.ac.kr \\ ${ }^{3}$ Institute of Astronomy and Department of Physics, National Tsing Hua University, Hsinchu, Taiwan \\ ${ }^{4}$ I. E. Tamm Theoretical Physics Division of P. N. Lebedev Institute of Physics, Leninskii pr. 53, 119991 Moscow, Russia \\ Received 2014 March 16; accepted 2014 May 21; published 2014 June 6
}

\begin{abstract}
In re-analyzing the archival Chandra data of the globular cluster 47 Tucanae, we have detected a new diffuse X-ray emission feature within the half-mass radius of the cluster. The spectrum of the diffuse emission can be described by a power-law model plus a plasma component with photon index $\Gamma \sim 1.0$ and plasma temperature $k T \sim 0.2 \mathrm{keV}$. While the thermal component is apparently uniform, the non-thermal contribution falls off exponentially from the core. The observed properties could possibly be explained in the context of multiple shocks resulting from the collisions among the stellar wind in the cluster and the inverse Compton scattering between the pulsar wind and the relic photons.
\end{abstract}

Key words: globular clusters: individual (47 Tucanae) - pulsars: general - X-rays: stars

Online-only material: color figures

\section{INTRODUCTION}

Globular clusters (GCs) are gravitationally bound dense systems hosting a very old stellar population of our Galaxy. Due to the high stellar densities in their cores, GCs are efficient in producing compact binaries through dynamical interactions (Pooley \& Hut 2006; Pooley et al. 2003; Hui et al. 2010). As millisecond pulsars (MSPs) are descendants of low-mass X-ray binaries (Alpar et al. 1982), GCs are expected to be efficient factories of MSPs.

In the high-energy regime, the Large Area Telescope on board the Fermi Gamma-ray Space Telescope has detected $\mathrm{GeV} \gamma$-rays from a number of Galactic GCs (Abdo et al. 2009a, 2010; Kong et al. 2010; Tam et al. 2011), confirming GCs as a class of $\gamma$-ray emitters. There are two possible explanations for the origin of $\gamma$-ray emission from GCs. In one scenario, the emission can be interpreted as the collective magnetospheric emission from the whole MSP population in the GC (e.g., Venter \& de Jager 2008). Indeed, the $\gamma$-ray spectra of these detected GCs are reminiscent of those measured from the Galactic MSP population (e.g., Abdo et al. 2009b) as well as the individual MSPs detected in GCs NGC 6624 (Freire et al. 2011) and M28 (Wu et al. 2013; Johnson et al. 2013). On the other hand, pulsar wind from the MSPs could also contribute to the observed $\gamma$-rays via the inverse Compton scattering (ICS) between relativistic pulsar wind leptons and background soft photons (Bednarek \& Sitarek 2007; Cheng et al. 2010; Hui et al. 2011). The ICS between these wind particles and the relic photons can also result in a diffuse X-ray structure (Cheng et al. 2010). Searching for this predicted extended feature in X-rays can help constrain the pulsar wind model of $\gamma$-ray GCs.

Hui et al. (2009) has searched for diffuse X-ray emission from the core regions of $10 \mathrm{GCs}$ using Chandra archival data, enabling unresolved X-ray emission to be found for four clusters, which was attributed to unresolved faint point source populations in the cores of the clusters. On the other hand, Eger et al. (2010) have detected diffuse X-ray emission associated with Terzan 5 outside its half-mass radius. They found that the emission can be characterized by a power law (PL) with hard photon index $\Gamma \sim 1$ and is unlikely to be contributed by a population of unresolved point sources below the detection limit. The hard photon index was suggested to be a result of a non-thermal scenario by synchrotron radiation from energetics considerations. However, the limited statistics did not enable this study to draw a firm conclusion.

Motivated by the aforementioned discovery, Eger \& Domainko (2012) has systematically searched archival Chandra data for diffuse X-ray emission from several Galactic GCs that have been detected in $\gamma$-rays by Fermi. The authors excluded 47 Tuc in their analysis because less than half of the potential diffuse emission region (i.e., between one and three half-mass radius $r_{\mathrm{hm}}$ ) is covered by the field of view (FoV) in the adopted data sets.

A previous search for diffuse X-ray emission in the direction of 47 Tuc identified an extended feature beyond its half-mass radius and in the direction of the proper motion of the cluster (Okada et al. 2007). However, such a feature was later confirmed to be not physically associated with 47 Tuc (Yuasa et al. 2009). While these studies were focused on an individual extended feature at a location offset from the center of 47 Tuc, we are interested in searching for an isotropic diffuse X-ray component associated with this GC.

Being a nearby GC at $4.5 \mathrm{kpc}$ (Harris 1996) and a host of a large MSP population, 47 Tuc is one of the promising targets to search for the theoretically predicted diffuse X-ray feature (cf. Cheng et al. 2010). Together with the large volume of archival X-ray data, this motivates a deep search for diffuse emission in an unexplored region outside the core radius $r_{c}$ of the cluster.

In this Letter, we report the results from searching for diffuse $\mathrm{X}$-ray emission associated with 47 Tuc in the region from $2 r_{c}$ up to $\sim 4^{\prime}$ from the center. This selected field can alleviate the problem of potential contamination by the unresolved faint source population (cf. Section 3) and allow a sufficiently large region for our search.

\section{DATA ANALYSIS AND RESULTS}

To search for faint diffuse X-ray emission from 47 Tuc, we made use of archival Chandra data and restricted our analysis 


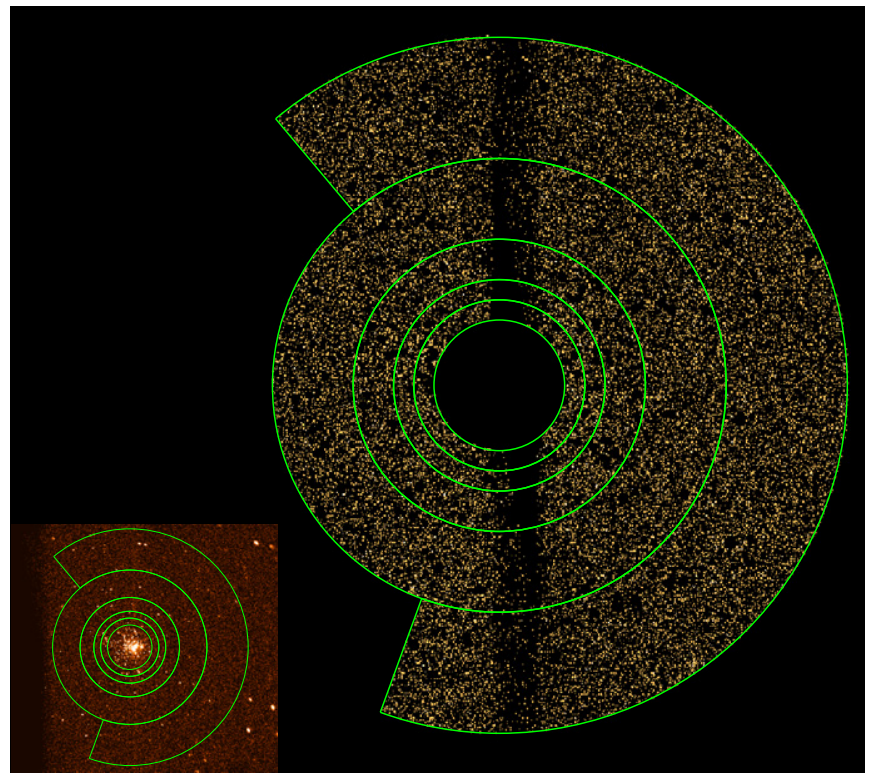

Figure 1. Combined $0.5-7.0 \mathrm{keV}$ events of all four observations after pointsource removal. The entire region-of-interest (RoI) is illustrated as an assembly of individual rings. The excluded rectangular regions to avoid pileup contamination is not shown. The inset shows the combined event file in the same energy band before point-source removal as an illustration of the position of the RoI in the GC.

(A color version of this figure is available in the online journal.)

to Advanced CCD Imaging Spectrometer (ACIS) data with an exposure $>10 \mathrm{ks}$. This limited us to four observations in 2002 (ObsID 2735, 2736, 2737, and 2738). The aim-points of all the four observations are on the S3 chip. We first reprocessed the data by using the CIAO (Version4.5) script chandra_repro with the calibration files CALDB (Version 4.5.5). Inspections of the $0.5-7.0 \mathrm{keV}$ light curves indicate that two observations (ObsID 2736 and 2738) suffered from prominent background flares. We subsequently performed the good time interval filtering by using the CIAO routine lc_clean(). Combining all the clean data resulted in an effective exposure of $\sim 238 \mathrm{ks}$.

Inspecting the reprocessed event files, signs of pileup effects caused by the bright sources in the core of the cluster were seen. We used the CIAO tool pileup_map and the associated conversion formulae to deduce the pileup fractions. ${ }^{5}$ It was found that the three brightest sources detected by Heinke et al. (2005), X7, X9 and X5, have pileup fractions $>5 \%$, with that of X7 exceeding $10 \%$. We excluded a rectangular region with a width of 0.5 centered on X7 and a length along the readout direction of the entire chip in subsequent spectrum extraction so as to avoid the contamination of the out-of-time events from the bright source.

To define the region-of-interest (RoI), we first chose the largest annular region in $2 r_{\mathrm{c}} \leqslant r \leqslant 2.5$ which is fully contained in the S3 chip. In order to fully utilize the FoV, we appended the aforementioned region with a truncated annular region extending to 3.75 (see Figure 1). The angular span of this region was chosen such that it lies entirely within the CCD chip in all four observations. The entire RoI in our study was defined by assembling all these adopted regions.

To effectively remove the resolved point sources in the RoI, we took the sky positions in the point source catalog by Heinke et al. (2005) and calculated the size of the Chandra point spread

\footnotetext{
See http://cxc.harvard.edu/csc/memos/files/Davis_pileup.pdf.
}

function (PSF) at $1.5 \mathrm{keV}$ with an enclosed-counts fraction of $96 \%$ at the positions of these point sources by using the psf Python module. Since Heinke et al. (2005) covers only sources within 2.79 of 47 Tuc, we ran wavdetect in the $0.5-7.0 \mathrm{keV}$ energy band to detect sources beyond this region. These sources were subsequently removed as aforementioned.

After removing all the resolved point sources and excluding the pileup-affected region, we extracted the spectra from individual observations within the entire RoI in the energy band of $0.5-7.0 \mathrm{keV}$.

We began our investigation with a spectral analysis of the $\mathrm{X}$-ray emission from the entire RoI. To tightly constrain the emission properties, we fitted the spectra extracted from all observations simultaneously. Since the RoI covers a large fraction of the S3 chip, there are limited source-free regions on the chip for extracting the background spectra. In addition, the peripheral region of the chip could possibly contain the contribution of diffuse X-ray emission that we are searching for. Therefore, we adopted the ACIS blank-sky event files as the background throughout our study. We followed the online analysis thread to tailor and reproject the blank-sky background files so as to match the observed data sets. ${ }^{6}$

To judge the background normalization, we have inspected the source and background spectra individually for each observation in 10-12 keV. In this band, the observed data are dominated by the particle background. Except for the data from ObsID 2738, the estimated background contributions for all other observations are $>99.7 \%$, which indicates that the particle background normalizations are legitimate. For ObsID 2738 , we noticed a residual background ( $\gtrsim 6 \%$ ) in this band. To minimize the systematic uncertainties, we excluded this data from all subsequent analysis. The response files used in this extended source analysis were computed by weighting the flux contribution from each pixel with the aid of a detector map.

For all spectral analyses, we fixed the column absorption at $N_{\mathrm{H}}=2.2 \times 10^{20} \mathrm{~cm}^{-2}$ which was deduced from the foreground reddening $E(B-V)=0.04$ (Harris 1996; Predehl \& Schmitt 1995; Cardelli et al. 1989). We first fitted the spectra using an absorbed PL model in the form of $K E^{-\Gamma}$, where the free parameters $K$ and $\Gamma$ are the normalization in units of photons $\mathrm{keV}^{-1} \mathrm{~cm}^{-2} \mathrm{~s}^{-1}$ at $1 \mathrm{keV}$ and the index of the photon flux, respectively. The best-fit yields a photon index of $\Gamma=4.48_{-0.16}^{+0.17}$. However, we noticed that the goodness of fit is not desirable. We also attempted to fit the data with a thermal plasma model which includes the contribution from the metal lines (XSPEC model: MEKAL). However, we found this model also results in a poor goodness of fit. On the other hand, by adding a MEKAL component to the PL model, a reasonable description of the observed data can be obtained $\left(\chi_{v}^{2}=1.15\right.$ for 415 d.o.f.). This composite model yields a photon index of $\Gamma=0.96_{-0.29}^{+0.34}$ and a plasma temperature of $k T=0.19 \pm 0.01$. An $F$-test indicates that the PL+MEKAL model is favored over a single PL or a single MEKAL model at a confidence level $>7 \sigma$. The energy spectrum of the entire RoI with the PL+MEKAL model fit is shown in Figure 2. All the spectral results are summarized in Table 1.

In order to estimate the contribution from unresolved point sources in the RoI, we constructed the cumulative luminosity function by using the luminosities of the sources within $r_{c}$ in $0.5-6 \mathrm{keV}$ reported by Heinke et al. (2005). Fitting the distribution with a functional form of $N\left(>L_{X}\right)=N_{0} L_{X}^{\alpha}$, we

\footnotetext{
6 http://cxc.harvard.edu/ciao/threads/acisbackground/
} 
Table 1

Spectral Fitting Results of the Entire RoI

\begin{tabular}{|c|c|c|c|c|c|c|}
\hline Model & $\chi_{v}^{2}$ (d.o.f.) & Norm $^{\mathrm{a}}$ & $\Gamma$ & $\begin{array}{c}k T^{\mathrm{b}} \\
(\mathrm{keV})\end{array}$ & $\begin{array}{c}L_{\mathrm{PL}}{ }^{\mathrm{c}} \\
\left(10^{32} \mathrm{erg} \mathrm{s}^{-1}\right)\end{array}$ & $\begin{array}{c}L_{\text {MEKAL }}{ }^{\mathrm{d}} \\
\left(10^{32} \mathrm{erg} \mathrm{s}^{-1}\right.\end{array}$ \\
\hline $\mathrm{PL}$ & $1.25(417)$ & $1.95_{-0.13}^{+0.13}$ & $4.48_{-0.16}^{+0.17}$ & $\cdots$ & $1.70_{-0.07}^{+0.06}$ & $\ldots$ \\
\hline MEKAL & $1.37(417)$ & $4.49_{-0.26}^{+0.26}$ & $\ldots$ & $0.20_{-0.01}^{+0.01}$ & $\ldots$ & $1.43_{-0.06}^{+0.06}$ \\
\hline PL+MEKAL & $1.15(415)$ & $0.90_{-0.17}^{+0.20} / 4.38_{-0.27}^{+0.27}$ & $0.96_{-0.29}^{+0.34}$ & $0.19_{-0.01}^{+0.01}$ & $2.36_{-0.48}^{+0.50}$ & $1.26_{-0.08}^{+0.07}$ \\
\hline
\end{tabular}

Notes.

a Normalization parameter of the PL/MEKAL model/component. It is $10^{-5}$ photons cm $\mathrm{cm}^{-2} \mathrm{~s}^{-1} \mathrm{keV}^{-1}$ at $1 \mathrm{keV}^{-}$

for a PL model. It is equal to $\left(10^{-14} / 4 \pi D^{2}\right) \int n_{\mathrm{e}} n_{\mathrm{H}} d V$ for a MEKAL model, where $D$ is taken to be $4.5 \mathrm{kpc}$.

b Plasma temperature.

${ }^{c}$ Integrated luminosity of the PL component in the energy band $0.5-7.0 \mathrm{keV}$.

${ }^{\mathrm{d}}$ Same as (c) but for the MEKAL component.

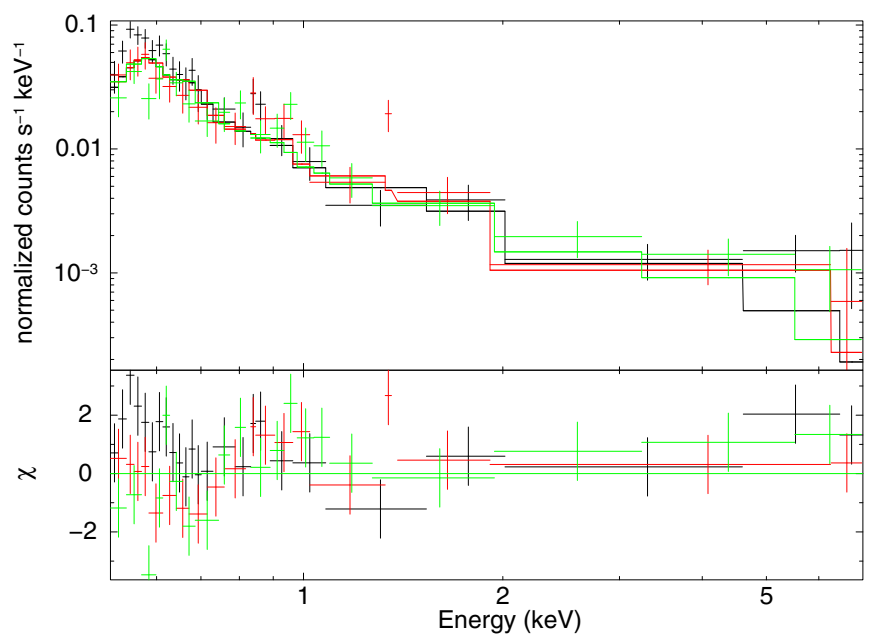

Figure 2. Upper panel: spectrum of the unresolved X-rays in the RoI, fitted by an absorbed power-law model plus a MEKAL plasma component. Lower panel: contributions to the $\chi^{2}$ fit statistic are shown.

(A color version of this figure is available in the online journal.)

obtained $\alpha=-0.55 \pm 0.01$ and $\log N_{0}=18.26 \pm 0.19$. With a putative detection limit of $8 \times 10^{29} \mathrm{erg} \mathrm{s}^{-1}$ (Heinke et al. 2005), we integrated the source density in the $0.5-6.0 \mathrm{keV}$ energy range to obtain a luminosity of $1 \times 10^{32} \mathrm{erg} \mathrm{s}^{-1}$ within $r_{\mathrm{c}}$. To estimate their contribution to our RoI, we adopted a radial source density with the form of:

$$
s(r)=s_{0}\left[1+\left(\frac{r}{r_{\mathrm{c}}}\right)^{2}\right]^{(1-3 q) / 2},
$$

where $s_{0}$ is a normalization factor and $q=1.63$ is the best-fit value obtained by Heinke et al. (2005). The expected luminosity of the unresolved point sources can then be estimated by $10^{32}\left(\int_{2 r_{\mathrm{c}}}^{3 !} s 35 s(r) d r / \int_{0}^{r_{\mathrm{c}}} s(r) d r\right) \mathrm{erg} \mathrm{s}^{-1} \approx 10^{31} \mathrm{erg} \mathrm{s}^{-1}$. To compare with this value, we calculated the measured luminosity in the same energy range to be $\left(3.82_{-0.30}^{+0.31}\right) \times 10^{32} \mathrm{erg} \mathrm{s}^{-1}$ for the PL+MEKAL model. Therefore, we conclude that the contribution from unresolved point sources to the observed emission is negligible.

To quantify the distribution of the diffuse X-ray emission as an angular distance from the center of the cluster, we divided the RoI into five concentric annular regions (rings). The sizes of each ring were chosen to obtain the spectral parameters with comparable relative errors in each ring. The fitting results of all rings are summarized in Table 2 . To obtain a surface brightness profile, we integrated the best-fit models for each ring over the

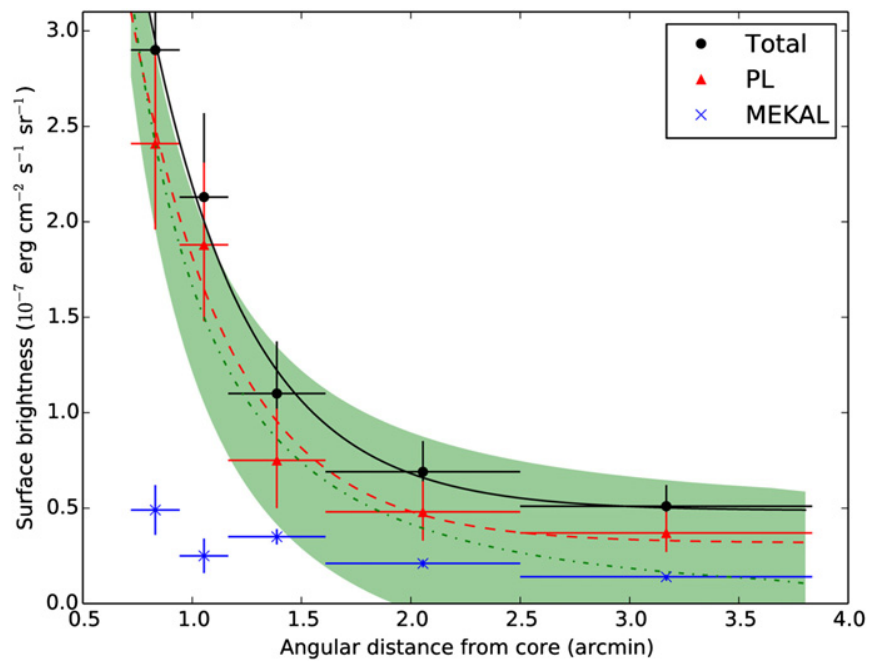

Figure 3. Unabsorbed diffuse X-ray surface brightness profile in the $0.5-7.0 \mathrm{keV}$ band (black circles). Red triangles and blue crosses represent the brightness of the PL component and the MEKAL component, respectively. The black solid line and the red dashed line show, respectively, the best-fit exponential profiles of the total brightness and the PL component. The green dot-dashed line denotes the stellar density profile from Michie (1963) and Kuranov \& Postnov (2006). It is scaled to match the first data point of the PL component and the uncertainty of this profile due to the error of the matched data point is shown by the greenshaded region.

(A color version of this figure is available in the online journal.)

energy band $0.5-7.0 \mathrm{keV}$ and divided the unabsorbed flux by the corresponding solid angle of the extraction region. The solid angles were computed with the exclusion of the PSF subtraction regions of point sources and the bad pixels. The brightness profile is plotted in Figure 3 in which the total surface brightness shows an exponential fall off. For a more detailed investigation, we disentangled the thermal and non-thermal contributions as shown in Figure 3. While the non-thermal X-rays follow an exponential fall off that conforms with the stellar density profile of 47 Tuc (green dot-dashed line in Figure 3), the plasma component is apparently uniform.

To quantify the structure of the diffuse emission, we fitted the function $S(r)=S_{0} \exp \left(-r / r_{\text {falloff }}\right)+C$ to the brightness profile, where $S_{0}$ is a normalization parameter, $r_{\text {falloff }}$ is the characteristic fall-off distance, and $C$ is the brightness at the tail. The best-fit for the total brightness yields $r_{\text {falloff }}=0.46 \pm 0.08$ and that for the PL component gives $r_{\text {falloff }}=0.45 \pm 0.15$, which are compatible within uncertainties. On the other hand, the best-fit constant levels for the total brightness and the PL component are found to be $C=(4.9 \pm 1.2) \times 10^{-8} \mathrm{erg} \mathrm{cm}^{-2} \mathrm{~s}^{-1} \mathrm{sr}^{-1}$ and $C=(3.2 \pm 2.1) \times 10^{-8} \mathrm{erg} \mathrm{cm}^{-2} \mathrm{~s}^{-1} \mathrm{sr}^{-1}$, respectively. 
Table 2

Spectral Fitting Results of Individual Rings by Using the PL+MEKAL Model

\begin{tabular}{llllllll}
\hline \hline Ring & $r_{\min }{ }^{\mathrm{a}}$ & $r_{\max }{ }^{\mathrm{b}}$ & $k T(\mathrm{keV})$ & \multicolumn{1}{c}{$\Gamma$} & $S_{\mathrm{PL}}{ }^{\mathrm{c}}$ & $S_{\mathrm{MEKAL}}{ }^{\mathrm{d}}$ & $S_{\text {total }}{ }^{\mathrm{e}}$ \\
\hline 1 & 0.72 & 0.9425 & $0.21 \pm 0.03$ & $0.77_{-0.32}^{+0.33}$ & $2.41_{-0.45}^{+0.48}$ & $0.49 \pm 0.13$ & $2.90_{-0.47}^{+0.50}$ \\
2 & 0.9425 & 1.165 & $0.17_{-0.07}^{+0.04}$ & $0.90_{-0.32}^{+0.33}$ & $1.88_{-0.40}^{+0.43}$ & $0.25 \pm 0.09$ & $2.13_{-0.41}^{+0.44}$ \\
3 & 1.165 & 1.61 & $0.25 \pm 0.02$ & $0.47_{-0.61}^{+0.62}$ & $0.75_{-0.25}^{+0.27}$ & $0.35 \pm 0.04$ & $1.10_{-0.25}^{+0.27}$ \\
4 & 1.61 & 2.5 & $0.18 \pm 0.01$ & $0.36_{-0.55}^{+0.54}$ & $0.48_{-0.15}^{+0.16}$ & $0.21 \pm 0.02$ & $0.69_{-0.15}^{+0.16}$ \\
5 & 2.5 & 3.835 & $0.18_{-0.02}^{+0.01}$ & $0.85_{-0.40}^{+0.34}$ & $0.37_{-0.10}^{+0.11}$ & $0.14_{-0.02}^{+0.01}$ & $0.51_{-0.10}^{+0.11}$ \\
\hline
\end{tabular}

Notes.

a Inner radius of the annular region in arcminutes.

b Outer radius of the annular region in arcminutes.

c Surface brightness for the PL component in $10^{-7} \mathrm{erg} \mathrm{cm}^{-2} \mathrm{~s}^{-1} \mathrm{sr}^{-1}$ over the energy range of $0.5-7.0 \mathrm{keV}$.

d Same as (c) but for the MEKAL component.

e Total surface brightness as a sum of (c) and (d).

\section{SUMMARY AND DISCUSSION}

In examining the archival Chandra data, we uncovered a faint extended X-ray feature in the region from $2 r_{c}$ to $\sim 4^{\prime}$ from the center of 47 Tuc. Figure 3 shows that the unabsorbed diffuse $\mathrm{X}$-rays in the $0.5-7.0 \mathrm{keV}$ band consist of two components, i.e., an exponential fall-off non-thermal component plus a uniform thermal component. The spectrum of the non-thermal component is very hard with a photon index $\Gamma \sim 1$. The surface brightness of this component apparently correlates with the point source distribution (i.e., Equation (1)) very well. The profiles of both extended feature and the radio point source density fall by $\sim 90 \%$ from $1^{\prime}$ to $2^{\prime}$ and $\sim 98 \%$ from $1^{\prime}$ to $3^{\prime}$.

It is known that stellar winds of massive stars are clumpy and non-thermal emission has been observed (cf. Feldmeier 2001 for a general review). In the core of a GC, collisions among stellar winds from high stellar density region could create multiple shocks that resemble the situation with stellar wind from massive stars. The electron distribution in multi-shock regions is known to have a very hard distribution $\left(d N_{e} / d E_{e} \sim E_{e}^{-1}\right)$ (Bykov \& Fleishman 1992; Bykov \& Toptygin 1993). For a PL distribution of electrons, $\left(d N_{e} / d E_{e}\right) \propto E_{e}^{-\alpha}$, the radiation via either synchrotron radiation or ICS will have a photon index of $\Gamma=(\alpha+1 / 2)$. Hence, a very hard X-ray spectrum is expected from the multi-shock regions.

To estimate the shock radius $R_{\mathrm{sh}}$, we used Equation (5) of Weaver et al. (1977): $R_{\mathrm{sh}} \sim\left(L_{w} \tau^{3} / \rho_{\mathrm{ISM}}\right)^{1 / 5}$, where $L_{w}$ and $\tau$ are the total wind power from the cluster and the expansion timescale, respectively. The wind power depends on the number of stars in the GC $N_{*}$, the mass-loss rate $\dot{M}$, and the wind velocity $v_{w}: L_{w} \sim N_{*} \dot{M} v_{w}^{2} / 2$. Taking the characteristic values of stellar wind, i.e., $\dot{M} \sim 10^{-14} M_{\odot} \mathrm{yr}^{-1}$ and $v_{w} \sim 500 \mathrm{~km} \mathrm{~s}^{-1}$, which gives $L_{w} \sim 10^{33}\left(N_{*} / 10^{6}\right) \mathrm{erg} \mathrm{s}^{-1}$. 47 Tuc has a finite proper motion at the order of $v_{p} \sim$ $150 \mathrm{~km} \mathrm{~s}^{-1}$ (cf. Okada et al. 2007). Assuming the shock maintains a spherical symmetry, the timescale can be estimated using Equation (64) of Weaver et al. (1977) which gives $\tau \sim 10^{12}\left(n_{\mathrm{ISM}} / 10^{-3} \mathrm{~cm}^{-3}\right)^{-1 / 2}\left(L_{w} / 10^{33} \mathrm{erg} \mathrm{s}^{-1}\right)^{1 / 2}$ $\left(v_{p} / 100 \mathrm{~km} \mathrm{~s}^{-1}\right)^{-5 / 2} \mathrm{~s}$, where $n_{\mathrm{ISM}} \sim \rho_{\mathrm{ISM}} / m_{p}$ and $m_{p}$ is the proton mass. Substituting all these into $R_{\text {sh }}$, the shock radius is found at an order of $\sim 10 \mathrm{pc}$. At $4.5 \mathrm{kpc}$, this corresponds to an angular size of $\sim 8^{\prime}$. Therefore the total stellar wind coming out the core of the GC can form a shock with the ISM at a distance much larger than $r_{c}$.

Consequently, the radiation away from the core should consist of two components, i.e., the non-thermal component emitted from non-thermal electrons accelerated in the diffuse shock region, whose energy index is 2-3 (Bell 1978), and the thermal component emitted from the shock-heated plasma. The temperature behind the shock is estimated to be $T \sim 3 \mu V_{\mathrm{sh}}^{2} / 16 k$ for an adiabatic shock (Weaver et al. 1977), where the shock velocity $V_{\mathrm{sh}}$ is estimated by $V_{\mathrm{sh}} \sim d R_{\mathrm{sh}} / d t \sim 3 R_{\mathrm{sh}} / 5 \tau \sim$ $3 \times 10^{7} \mathrm{~cm} \mathrm{~s}^{-1}$. Hence $T \sim 2 \times 10^{6} \mathrm{~K}$ which is consistent with the observed value. We can also estimate the thermal $\mathrm{X}$-ray luminosity from thermal bremsstrahlung which is a function of local plasma density, plasma temperature, and the total volume of interest (Rybicki \& Lightman 1979): $L_{x} \sim$ $10^{-27} n^{2} T^{1 / 2} V$. To estimate the plasma density, we can utilize the normalization parameter deduced from the best-fit plasma model $\int n_{e} n_{H} d V \sim 10^{55} \mathrm{~cm}^{-3}$. Assuming $n_{e} \sim n_{H}$, we obtain $n_{e} \sim 0.03\left(V / V_{\mathrm{RoI}}\right)^{-1 / 2} \mathrm{~cm}^{-3}$, where we adopted the radius of the RoI as $r_{\text {RoI }} \sim 5 \mathrm{pc}$. We noticed that this estimate is less than the electron density $\left(n_{e} \sim 0.07 \mathrm{~cm}^{-3}\right)$ obtained by Freire et al. (2001). The underestimation can stem from ignoring the metal content in the cluster. Adopting $T \sim 2 \times 10^{6} \mathrm{~K}, n \sim 0.07 \mathrm{~cm}^{-3}$ and $V \sim 2 \times 10^{58} \mathrm{~cm}^{3}$, the thermal bremsstrahlung luminosity is on the order of $L_{x} \sim 10^{32} \mathrm{erg} \mathrm{s}^{-1}$ which agrees with the observed value.

Apart from the shock produced by the stellar wind and ISM, we also considered other possible contribution to the non-thermal component. Cheng et al. (2010) showed that ICS between the background soft photon fields and the relativistic electrons in the pulsar wind can contribute to the $\gamma$-ray from GCs. They predict that a uniform X-ray component should exist due to ICS between the relic photons and the pulsar wind relativistic electrons. The X-ray flux is proportional to the $\gamma$-ray flux as $F_{x}(5 \mathrm{keV}) \approx\left(5 \mathrm{keV} / 8 \gamma_{w 5}^{2} \mathrm{MeV}\right)^{0.5}\left(w_{\text {relic }} / w_{\text {soft }}\right) F_{\gamma}^{\text {obs }}$, where $\gamma_{w 5}$ is the Lorentz factor of the pulsar wind in units of $10^{5}, F_{\gamma}^{\text {obs }}$ is the observed $\gamma$-ray energy flux in the GeV regime, $L_{34}$ is the typical spin-down power of MSP in units of $10^{34} \mathrm{erg} \mathrm{s}^{-1}$, and $f_{e^{ \pm}} \sim 30$ is the energy ratio between electrons and protons. The Lorentz factor of the pulsar wind is estimated as $\gamma_{w}=2 \times 10^{5} f_{e^{ \pm}}^{-1} L_{34}^{1 / 2} \sim 6 \times 10^{3}$. Taking the ratio between the relic photon energy density $w_{\text {relic }}$ and soft photon energy density $w_{\text {soft }}$ to be $\left(w_{\text {relic }} / w_{\text {soft }}\right) \sim 1 / 2$ (Cheng et al. 2010) and $F_{\gamma}^{\text {obs }} \sim 10^{-11} \mathrm{erg} \mathrm{cm}^{-2} \mathrm{~s}^{-1}$ (Abdo et al. 2010), we obtain $F_{x}(5 \mathrm{keV}) \sim 3 \times 10^{-12} \mathrm{erg} \mathrm{cm}^{-2} \mathrm{~s}^{-1}$. Since the $\gamma$-ray emission from 47 Tuc is consistent with a point source as observed by Fermi, its emission radius must be less than the PSF of the telescope (i.e., $\lesssim 30^{\prime}$ ). The corresponding inverse Compton (IC) X-ray luminosity emitted from $5^{\prime}$ is given 
by $L_{x}\left(r<5^{\prime}\right) \sim F_{x} 4 \pi D^{2}(5 / 30)^{2} \sim 3 \times 10^{32}$ erg s${ }^{-1}$, where $D \sim 5 \mathrm{kpc}$ is the distance to 47 Tuc, which is consistent with the observed non-thermal luminosity given in Table 1.

Follow-up investigations of the nature of the extended X-ray feature of 47 Tuc are encouraged. Weaver et al. (1977) suggested that a large number of emission lines should be emitted from the shock-heated plasma. Deep X-ray observation with high spectral resolution is required to resolve them. Based on the IC model, radio flux from the pulsar wind should be also correlated with the $\gamma$-ray flux (Cheng et al. 2010); future radio observations with higher resolution and sensitivity can also help to constrain the model.

C.Y.H. is supported by the National Research Foundation of Korea through grant 2011-0023383. A.K.H.K. is supported by the National Science Council of the Republic of China (Taiwan) through grant NSC100-2628-M-007-002-MY3 and NSC1002923-M-007-001-MY3. P.H.T. is supported by the National Science Council of the Republic of China (Taiwan) through grant NSC101-2112-M-007-022-MY3. K.S.C. is supported by a GRF grant of HK Government under HKU7009 11P. V.A.D. acknowledges support from the RFFI grant 12-02-00005.

\section{REFERENCES}

Abdo, A. A., Ackermann, M., Ajello, M., et al. 2009a, Sci, 325, 845

Abdo, A. A., Ackermann, M., Ajello, M., et al. 2009b, Sci, 325, 848
Abdo, A. A., Ackermann, M., Ajello, M., et al. 2010, A\&A, 524, A75

Alpar, M. A., Cheng, A. F., Ruderman, M. A., \& Shaham, J. 1982, Natur, 300,728

Bednarek, W., \& Sitarek, J. 2007, MNRAS, 377, 920

Bell, A. R. 1978, MNRAS, 182, 147

Bykov, A. M., \& Fleishman, G. D. 1992, MNRAS, 255, 269

Bykov, A. M., \& Toptygin, I. N. 1993, PhyU, 36, 1020

Cardelli, J. A., Clayton, G. C., \& Mathis, J. S. 1989, ApJ, 345, 245

Cheng, K. S., Chernyshov, D. O., Dogiel, V. A., et al. 2010, ApJ, 723, 1219

Eger, P., \& Domainko, W. 2012, A\&A, 540, 17

Eger, P., Domainko, W., \& Clapson, A.-C. 2010, A\&A, 513, 66

Feldmeier, A. 2001, PhD thesis, Potsdam Univ.

Freire, P. C., Kramer, M., Lyne, A. G., et al. 2001, ApJL, 557, L105

Freire, P. C. C., Abdo, A. A., Ajello, M., et al. 2011, Sci, 334, 1107

Harris, W. E. 1996, AJ, 112, 1487

Heinke, C. O., Grindlay, J. E., Edmonds, P. D., et al. 2005, ApJ, 625, 796

Hui, C. Y., Cheng, K. S., \& Taam, R. E. 2009, ApJ, 700, 1233

Hui, C. Y., Cheng, K. S., \& Taam, R. E. 2010, ApJ, 714, 1149

Hui, C. Y., Cheng, K. S., Wang, Y., et al. 2011, ApJ, 726, 100

Johnson, T. J., et al. 2013, ApJ, 778, 106

Kong, A. K. H., Hui, C. Y., \& Cheng, K. S. 2010, ApJL, 712, L36

Kuranov, A. G., \& Postnov, K. A. 2006, AstL, 32, 393

Michie, R. W. 1963, MNRAS, 125, 127

Okada, Y., Kokubun, M., Yuasa, T., \& Makishima, K. 2007, PASJ, 59, 727

Pooley, D., \& Hut, P. 2006, ApJL, 646, L143

Pooley, D., Lewin, W. H. G., Anderson, S. F., et al. 2003, ApJL, 591, L131

Predehl, P., \& Schmitt, J. H. M. M. 1995, A\&A, 293, 889

Rybicki, G.B., \& Lightman, A.L. 1979, Radiative Processes in Astrophysics (New York: Wiley)

Tam, P. H. T., Kong, A. K. H., Hui, C. Y., et al. 2011, ApJ, 729, 90

Venter, C., \& de Jager, O. C. 2008, ApJL, 680, L125

Weaver, R., McCray, R., \& Castor, J. 1977, ApJ, 218, 377

Wu, J. H. K., Hui, C. Y., Wu, E. M. H., et al. 2013, ApJL, 765, L47

Yuasa, T., Nakazawa, K., \& Makishima, K. 2009, PASJ, 61, 1107 